\title{
CpG Island Methylation in Sessile Serrated Adenoma/Polyp of the Colorectum: Implications for Differential Diagnosis of Molecularly High-Risk Lesions among Non-dysplastic Sessile Serrated Adenomas/Polyps
}

\author{
Ji Ae Lee ${ }^{1} \cdot$ Hye Eun Park ${ }^{1}$ \\ Seung-Yeon $\mathrm{Yoo}^{1} \cdot$ Seorin Jeong ${ }^{2}$ \\ Nam-Yun $\mathrm{Cho}^{2} \cdot$ Gyeong Hoon Kang ${ }^{1,2}$ \\ Jung Ho Kim ${ }^{1,2}$
}

'Department of Pathology, Seoul National University Hospital, Seoul National University College of Medicine, Seoul; ' 2 Laboratory of Epigenetics, Cancer Research Institute, Seoul National University College of Medicine, Seoul, Korea

Received: February 16, 2019

Revised: March 9, 2019

Accepted: March 12, 2019

\section{Corresponding Author}

Jung Ho Kim, MD, PhD

Department of Pathology, Seoul National University Hospital, 101 Daehak-ro, Jongno-gu, Seoul 03080, Korea

Tel: +82-2-2072-2828

Fax: +82-2-743-5530

E-mail: junghokim@snuh.org

\begin{abstract}
Background: Although colorectal sessile serrated adenomas/polyps (SSA/Ps) with morphologic dysplasia are regarded as definite high-risk premalignant lesions, no reliable grading or risk-stratifying system exists for non-dysplastic SSA/Ps. The accumulation of $\mathrm{CpG}$ island methylation is a molecular hallmark of progression of SSA/Ps. Thus, we decided to classify non-dysplastic SSA Ps into risk subgroups based on the extent of $\mathrm{CpG}$ island methylation. Methods: The $\mathrm{CpG}$ island methylator phenotype (CIMP) status of 132 non-dysplastic SSA/Ps was determined using eight CIMP-specific promoter markers. SSA/Ps with CIMP-high and/or MLH1 promoter methylation were regarded as a high-risk subgroup. Results: Based on the CIMP analysis results, methylation frequency of each CIMP marker suggested a sequential pattern of $\mathrm{CpG}$ island methylation during progression of SSA/P, indicating MLH1 as a late-methylated marker. Among the 132 non-dysplastic SSA/Ps, 34 (26\%) were determined to be high-risk lesions (33 CIMP-high and 8 MLH1methylated cases; seven cases overlapped). All 34 high-risk SSA/Ps were located exclusively in the proximal colon $(100 \%, p=.001)$ and were significantly associated with older age ( $\geq 50$ years, $100 \% ; p=.003$ ) and a larger histologically measured lesion size (>5 mm, 100\%; $p=.004)$. In addition, the high-risk SSA/Ps were characterized by a relatively higher number of typical base-dilated serrated crypts. Conclusions: Both CIMP-high and MLH1 methylation are late-step molecular events during progression of SSA/Ps and rarely occur in SSA/Ps of young patients. Comprehensive consideration of age ( $\geq 50$ ), location (proximal colon), and histologic size ( $>5 \mathrm{~mm}$ ) may be important for the prediction of high-risk lesions among non-dysplastic SSA/Ps.
\end{abstract}

Key Words: Colorectal neoplasms; DNA methylation; Serrated lesion; Serrated pathway; Serrated polyp
It is widely known that there are two morphologic multistep colorectal carcinogenesis pathways: the conventional pathway and the serrated pathway. ${ }^{1,2}$ In the conventional pathway, colorectal carcinomas (CRCs) develop through premalignant lesions, including tubular, tubulovillous, and villous adenomas, accounting for approximately $60 \%-80 \%$ of the CRCs, whereas in the serrated pathway, about $15 \%-35 \%$ of the CRCs progress from serrated precursor lesions. ${ }^{1,3,4}$ According to the latest (4th) edition of the World Health Organization (WHO) classification of tumors of the digestive system, serrated colorectal lesions are classified into three categories: hyperplastic polyps (HPs), sessile serrated adenomas/polyps (SSA/Ps), and traditional serrated adenomas (TSAs). ${ }^{5}$ Among the three subtypes of serrated lesions, SSA/Ps and TSAs are recognized as premalignant lesions, whereas
HPs are not regarded as direct precursors of carcinoma but rather as potential precursors of SSA/Ps or TSAs. ${ }^{5,6}$

$\mathrm{SSA} / \mathrm{Ps}$ are considered major precursors of the $\mathrm{CpG}$ island methylator phenotype-high (CIMP-high) CRCs. ${ }^{1,3,-8}$ Histologically, SSA/Ps are typically characterized by distorted crypt architecture, including horizontal extension (inverted T- or L-shape) and branching of the lumen at the crypt bases. ${ }^{5,6,9}$ Through genetic and epigenetic alterations, they can acquire cytologic dysplasia and ultimately progress toward carcinoma. The typical genetic and epigenetic characteristics of SSA/Ps are BRAF V600E mutation and $\mathrm{CpG}$ island hypermethylation, respectively.,

It has been suggested that risk factors to predict the progression of SSA/Ps are morphologic dysplasia, size of the polyp, and the number of serrated polyps. ${ }^{6,10}$ Based on these potential risk 
factors, endoscopic surveillance guidelines have generally recommended shorter surveillance interval for SSA/Ps with morphologic dysplasia, large polyp size ( $\geq 10 \mathrm{~mm})$, or multiple SSA/Ps or TSAs (3 or more). ${ }^{6}$ However, although SSA/Ps without dysplasia are more prevalent than SSA/Ps with dysplasia, there has been a lack of reliable grading or risk-stratifying system for non-dysplastic SSA/Ps. Therefore, identifying clinicopathologic factors correlated with underlying molecular alterations that indicate high-risk lesions would be important for establishing a precise risk-assessment system for non-dysplastic SSA/Ps.

The accumulation of $\mathrm{CpG}$ island methylation is known as a molecular hallmark of the progression of SSA/Ps. ${ }^{11,12}$ Thus, CIMP-high and MLH1 methylation status can be strong molecular indicators of the high potential of malignant change in SSA Ps. In this study, DNA methylation status of non-dysplastic SSA/Ps was analyzed using DNA extracted from the lesions, and non-dysplastic SSA/Ps were classified into two risk subgroups based on the CIMP status: high-risk (CIMP-high and/or MLH1 promoter hypermethylation) and low-risk (CIMP-low/negative) subgroups. We then aimed to investigate the differential clinicopathologic characteristics between high-risk and low-risk subgroups. Through this analysis, we expect to find clinicopathologic factors that can aid in the prediction of molecularly highrisk lesions among non-dysplastic SSA/Ps.

\section{MATERIALS AND METHODS}

\section{Tissue samples}

We initially reviewed a total of 275 colorectal polyps diagnosed with the term "sessile serrated adenoma" or "sessile serrated polyp," all of which had been removed by endoscopic mucosal resection or polypectomy between 2010 and 2012 at Seoul National University Hospital, Seoul, Korea. The recommendations proposed by an American expert panel in 2012 were used to diagnose SSA/Ps in our study. ${ }^{6}$ Of these 275 cases, 234 were determined to be morphologically definite SSA/Ps through independent microscopic review by three gastrointestinal pathologists (J.A.L., H.E.P., and J.H.K.) Finally, 133 cases were selected as potential pure forms of sporadic non-dysplastic SSA/Ps, based on the strict inclusion and exclusion criteria of this study, listed below:

- Inclusion criteria: presence of one or more typical distorted crypt for SSA/P (dilated and/or horizontally branched crypt base) in the lesion

- Exclusion criteria: satisfying one of the WHO diagnostic criteria for serrated polyposis, ${ }^{5}$ presence of TSA or conventional adenoma component in the lesion (mixed polyp), and presence of morphologic dysplasia in the lesion

This study was approved by the Institutional Review Board of Seoul National University Hospital (IRB No. 1804-109939). Under the condition of retrospective archival tissue collection and patient data anonymization, our study was exempted from the acquisition of informed consent from patients.

\section{Clinical data collection and histomorphometric analysis}

Clinical data, including the age and sex of patients, size and location of non-dysplastic SSA/Ps, and number and diagnosis of synchronous polyps, were collected by reviewing the electronic medical records. Sidedness of the location of each SSA/P was categorized into proximal or distal using splenic flexure as the cutoff site. Multiplicity of serrated lesions was determined by counting the total number of polyps diagnosed as HP, SSA/P, TSA or serrated polyp (unclassified), which were biopsied or resected during the same endoscopic evaluation. In addition to the endoscopically measured size of non-dysplastic SSA/Ps, we measured the maximum size of each SSA/P histologically using the Aperio ImageScope program (ver. 12.0.0., Leica Biosystems, Nussloch, Germany) (Fig. 1A). Other quantitative histomorphometric analyses, including the total number and the largest diameter of typical base-dilated serrated crypts in each SSA/P, were also performed using the Aperio ImageScope program (Fig. 1B, C).

\section{DNA methylation analysis}

DNA was extracted from formalin-fixed paraffin-embedded (FFPE) tissue blocks of 133 non-dysplastic SSA/Ps. After the extraction of genomic DNA, the DNA quantity of one of the 133 SSA/Ps was found to be insufficient for DNA experiments, and therefore, a total of 132 non-dysplastic SSA/Ps were finally subjected to DNA methylation analysis. Bisulfite modification and CIMP analysis of the $132 \mathrm{DNA}$ samples were conducted as previously described. ${ }^{13,14}$ For CIMP determination, methylation-specific quantitative polymerase chain reaction (PCR) analysis (MethyLight assay) was performed using eight CIMPspecific promoter markers (MLH1, CACNA1G, CDKN2A (p16), CRABP1, IGF2, NEUROG1, RUNX3, and SOCS1). A CIMP marker was defined as hypermethylated when the percentage of methylated reference (PMR) value was 4 or more. Samples were classified as CIMP-high if they showed $\mathrm{CpG}$ island hypermethylation in five or more CIMP markers, and as CIMP-low if one to four CIMP markers were hypermethylated. CIMP-negative indicated that there was no hypermethylated marker in the sample. The classification criteria, marker panel, and PMR cut- 


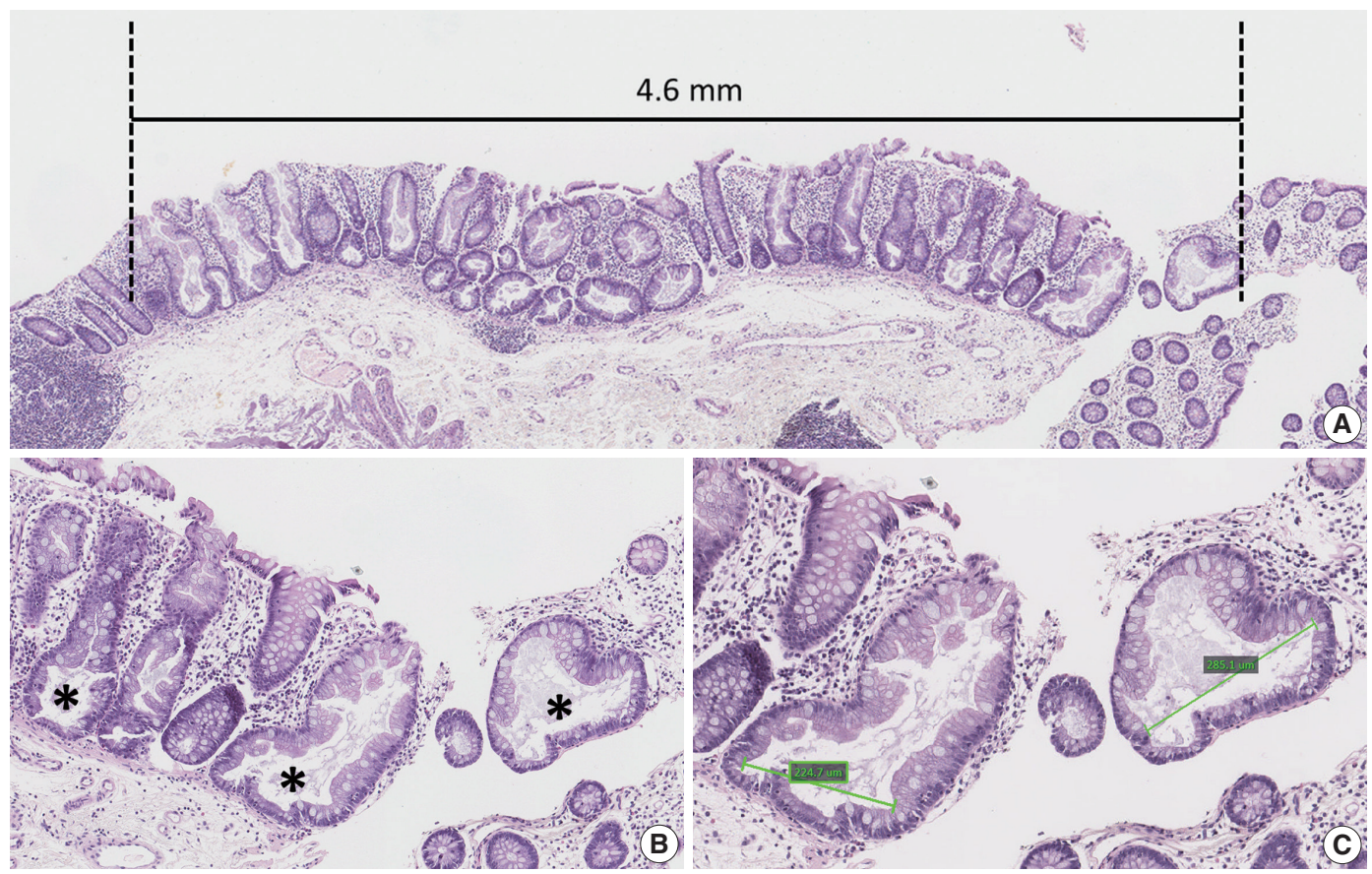

Fig. 1. Histomorphometric analyses used in this study. (A) A representative example for measurement of maximum size of a sessile serrated adenomas/polyp (SSA/P). (B) A representative example for counting of typical base-dilated serrated crypts. The total number of typical crypts in an SSAVP was used as a variable in this study. Asterisks indicate typical crypts. (C) A representative example for measurement of diameters of typical base-dilated serrated crypts. The largest diameter of typical crypts in an SSA/P was used as a variable in this study.

off value for CIMP determination used in this study were based on the methodology developed by Dr. Shuji Ogino that had been validated by previous studies. ${ }^{15-18}$ The methylation levels of long interspersed nucleotide element-1 (LINE-1) were also measured in 132 non-dysplastic SSA/Ps using pyrosequencing assay. The primers and PCR conditions were used as described previously. ${ }^{19}$

\section{Immunohistochemistry}

Immunohistochemistry (IHC) for MLH1 was performed on whole FFPE tissue sections of 132 non-dysplastic SSA/Ps using the Ventana Benchmark XT automated immunostainer (Roche, Basel, Switzerland) with a primary antibody against MLH1 (clone M1, Ventana RTU, Roche).

\section{Statistical analysis}

Statistical analyses in this study were performed using the IBM SPSS Statistics ver. 23 (IBM Corp., Armonk, NY, USA). Comparisons of categorical variables were conducted using the chi-square test or Fisher exact test. Comparisons of continuous variables were conducted using the Student's t test or MannWhitney U test. All p-values were two-sided, and the statistical significance was determined at $\mathrm{p}<.05$.

\section{RESULTS}

Developmental pattern of $\mathrm{CpG}$ island methylation in SSA/Ps Among 132 non-dysplastic SSA/Ps, the number of CIMPhigh, CIMP-low, and CIMP-negative cases were 33 (25\%), 73 (55\%), and 26 (20\%), respectively. First, to infer a developmental pattern of CpG island methylation during progression of SSA/ Ps, we performed in-depth analyses using methylation results of each CIMP marker in the 132 SSA/Ps (Fig. 2, Supplementary Fig. S1). Based on the number of concurrently methylated markers, eight CIMP markers were classified into three groups (Fig. 2A). Group A markers, including CRABP1, NEUROG1, and $C D K N 2 A(p 16)$, represented early-methylated markers in the serrated neoplasia pathway, because they could be methylated without any other concurrently methylated marker in SSA/Ps. In contrast, group C markers, including SOCS1 and MLH1, represented late-methylated markers, because they could not be methylated without at least two or three concurrently methylated markers (Fig. 2A, B). Group B markers, including CAC$N A 1 G$, IGF2, and RUNX3, could be methylated when only one other marker was methylated, but not when there was no other concurrently methylated marker (Fig. 2A). Thus, CACNA1G, $I G F 2$, and RUNX3 could be regarded as intermediately meth- 
ylated markers in the progression pathway of SSA/Ps (Fig. 2B). In addition, the characteristic sequential pattern of methylation of CIMP markers in SSA/Ps was closely associated with age of the patients. We further analyzed the percentages of methylationpositive cases for each CIMP marker in the 132 SSA/Ps, according to age subgroups (Supplementary Fig. S1). We found that

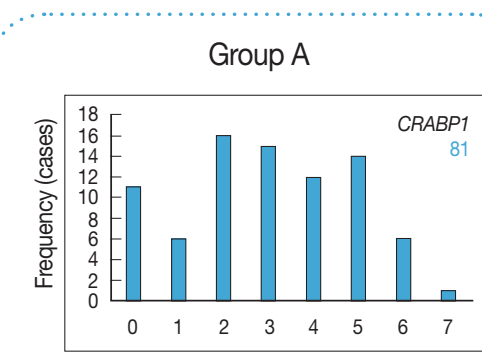

No. of concurrently methylated markers

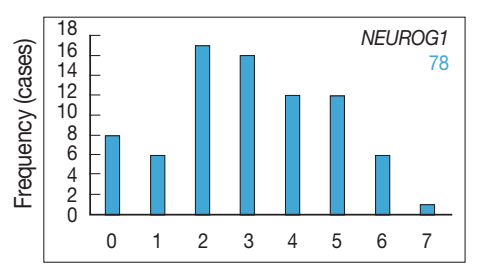

No. of concurrently methylated markers

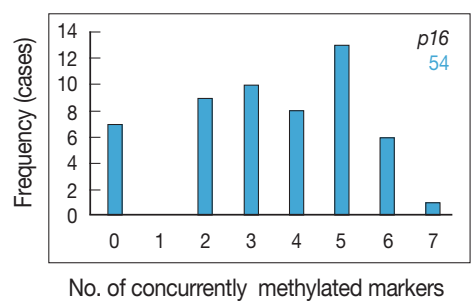

Group B
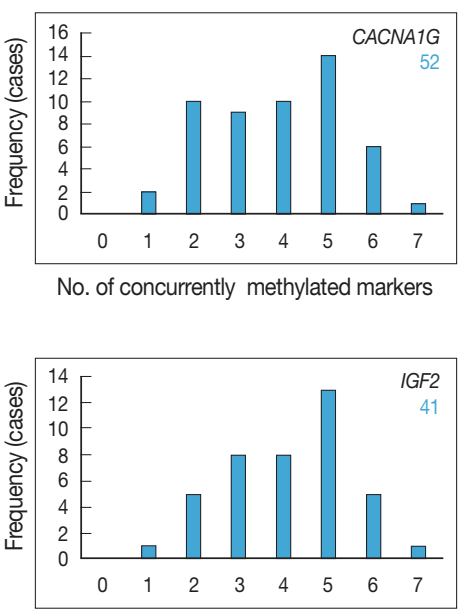

No. of concurrently methylated markers

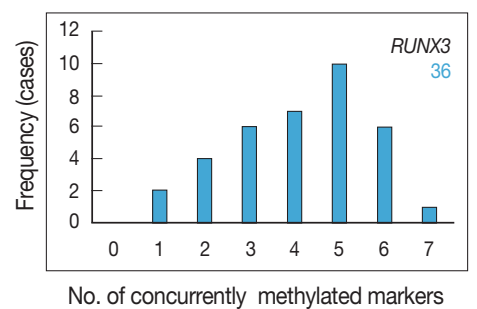

Group C

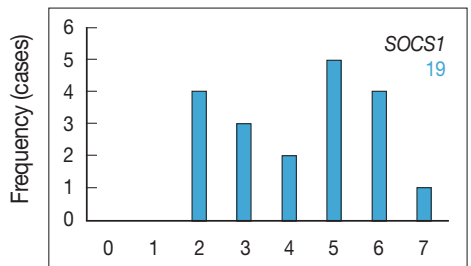

No. of concurrently methylated markers

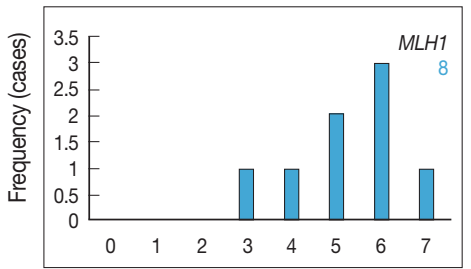

No. of concurrently methylated markers

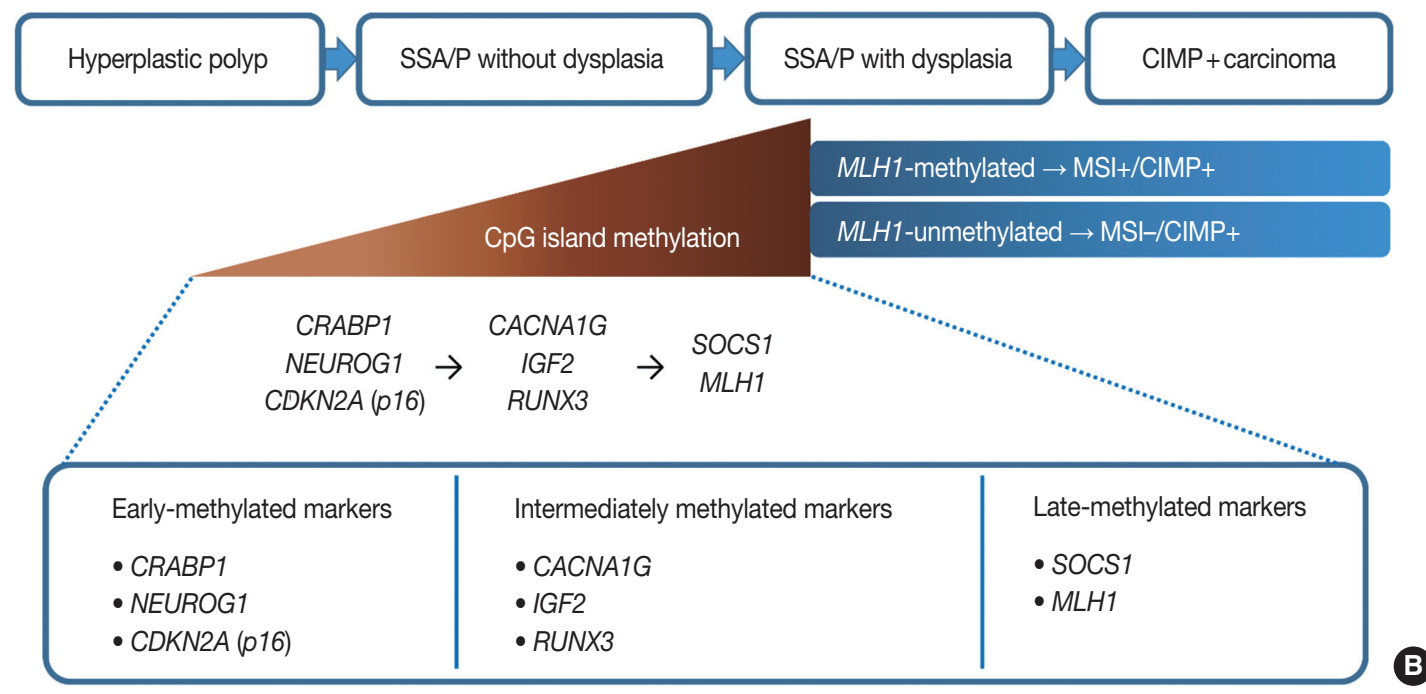

Fig. 2. An inference of developmental pattern of $\mathrm{CpG}$ island methylation in colorectal serrated neoplasia pathway. (A) Frequencies of methylation-positive cases for each CpG island methylator phenotype (CIMP) marker, based on the number of concurrently methylated markers in 132 non-dysplastic sessile serrated adenomas/polyps (SSA/Ps). Group A markers can be methylated independently and alone, whereas methylation of group C markers accompanies at least two or three other methylated markers. (B) A sequence model of methylation of CIMP markers during progression of SSAVP. 
CRABP1 and NEUROG1, the early-methylated markers, were methylated even in the younger age group $(<40)$ (Supplementary Fig. S1A, S1B), whereas SOCS1 and MLH1, the late-methylated markers, were not methylated before the age of 50 (Supplementary Fig. S1G, S1H).

\section{MLH1 expression status in MLH1-methylated SSA/Ps}

Eight of 132 non-dysplastic SSA/Ps (6\%) were determined to be MLH1-methylated. Using IHC, we evaluated the MLH1 protein expression status in eight $\mathrm{MLH1}$-methylated non-dysplastic SSA/Ps. Surprisingly, among the eight MLH1-methylated non-dysplastic SSA/Ps, there was no case with a complete loss of $\mathrm{MLH1}$ expression (Supplementary Fig. S2). To compare the differential features between non-dysplastic and dysplastic SSA/ Ps harboring $M L H 1$ methylation, we analyzed two additional cases confirmed as MLH1-methylated SSA/Ps with morphologic dysplasia (Supplementary Table S1). In contrast to the MLH1methylated non-dysplastic SSA/Ps, both these MLH1-methylated dysplastic SSA/Ps demonstrated a complete loss of MLH1 expression (Supplementary Table S1, Supplementary Fig. S2D). However, only one of the eight MLH1-methylated non-dysplastic SSA/Ps showed a partial loss of MLH1 expression (Supplementary Table S1, Supplementary Fig. S2B, S2C). The MLH1 promoter PMR value (26.3) for this MLH1-partial-deficient case was greater than that of the other MLH1-methylated nondysplastic SSA/Ps (5 to 12.9), but less than that of MLH1methylated dysplastic SSA/Ps (36.04 to 57.07) (Supplementary Table S1). Therefore, it could be interpreted that MLH1 methylation found in non-dysplastic SSA/Ps represents subrepressive, low-level methylation. It is also notable that $M L H 1$-methylated dysplastic SSA/Ps, as well as MLH1-methylated non-dysplastic SSA/Ps, were found exclusively in individuals aged 50 years or more (Supplementary Table S1).

\section{Clinicopathologic factors associated with high-risk subgroup of non-dysplastic SSA/Ps}

In this study, we defined the high-risk subgroup of SSA/Ps as lesions harboring CIMP-high and/or MLH1 promoter methylation. As mentioned above, 33 of 132 non-dysplastic SSA/Ps (25\%) were CIMP-high lesions, and eight of 132 non-dysplastic SSA/Ps (6\%) demonstrated MLH1 methylation. Seven of the eight MLH1-methylated SSA/Ps overlapped with CIMPhigh cases. Thus, among the 132 non-dysplastic SSA/Ps, 34 cases (26\%) were finally determined to be high-risk SSA/Ps, but the remaining 98 cases (74\%) were classified into low-risk SSA/Ps. Notably, the high-risk subgroup was characterized by a specific age group and lesion location (Table 1, Fig. 3). CIMP-high and MLH1 methylation were found only in individuals aged 50 years or more (Fig. 3A, Supplementary Table S1, Supplementary Fig. S1H). Moreover, high-risk SSA/Ps were located exclusively in the proximal colon, including cecum, ascending colon, hepatic flexure, and transverse colon (Fig. 3B), whereas low-risk SSA/Ps were distributed throughout the whole colorectum, although they were also enriched in the proximal colon (Fig. 3C). Collectively, the probability of high-risk SSA/Ps could be restricted to proximal colon-located SSA/Ps found in 50 or more-aged adults (Fig. 3D).

Differential clinicopathologic characteristics between highrisk and low-risk subgroups of the 132 non-dysplastic SSA/Ps are summarized in Table 1. In detail, high-risk SSA/Ps were significantly associated with older age $(\geq 50$ years old, $100 \%$; $\mathrm{p}=$ $.003)$, males $(82 \% ; \mathrm{p}=.015)$, proximal colonic location $(100 \%$; $\mathrm{p}=.001)$, larger histologically measured lesion size $(>5 \mathrm{~mm}$, $100 \% ; \mathrm{p}=.004)$, and higher number of typical base-dilated serrated crypts ( $\geq 3$ typical crypts, 94\%; $\mathrm{p}=.024)$. The mean histologically measured lesion size of high-risk SSA/Ps was significantly higher than that of low-risk SSA/Ps $(10.3 \mathrm{~mm}$ vs $8.4 \mathrm{~mm}$; $\mathrm{p}=$ .034) (Fig. 4A). High-risk SSA/Ps were found only in > 5-mmsized SSA/Ps, whereas a substantial number of low-risk SSA/Ps were 1- to 5-mm-sized lesions (Fig. 4B). The mean number of typical base-dilated crypts for high-risk lesions was also significantly higher than that of low-risk lesions (18.8 vs $10.7, \mathrm{p}=$ .031) (Fig. 4C). However, the distribution of number of typical crypts between high-risk and low-risk SSA/Ps was similar, except for a few high-risk lesions with a very high number of typical crypts (Fig. 4D).

Lastly, high-risk SSA/Ps were not significantly correlated with multiplicity of serrated lesions (Table 1, Supplementary Fig. S3), largest diameter of typical base-dilated crypts (Table 1, Supplementary Fig. S4), or the average LINE-1 methylation level (Table 1, Supplementary Fig. S5).

\section{DISCUSSION}

Until recently, number (many), size (large), anatomic site (rightsided colon), and morphologic dysplasia of SSA/Ps have been regarded as the potential risk factors for the progression of SSA/ Ps into carcinomas. ${ }^{6,10}$ However, among these potential risk factors, only morphologic dysplasia is based on strong molecular and pathologic evidence, such as high incidences of MLH1 deficiency, CIMP-high, and accompanying carcinomatous component in SSA/Ps with dysplasia. ${ }^{6,20,21}$ The other potential risk factors for malignant change of SSA/Ps, including number, size, and 


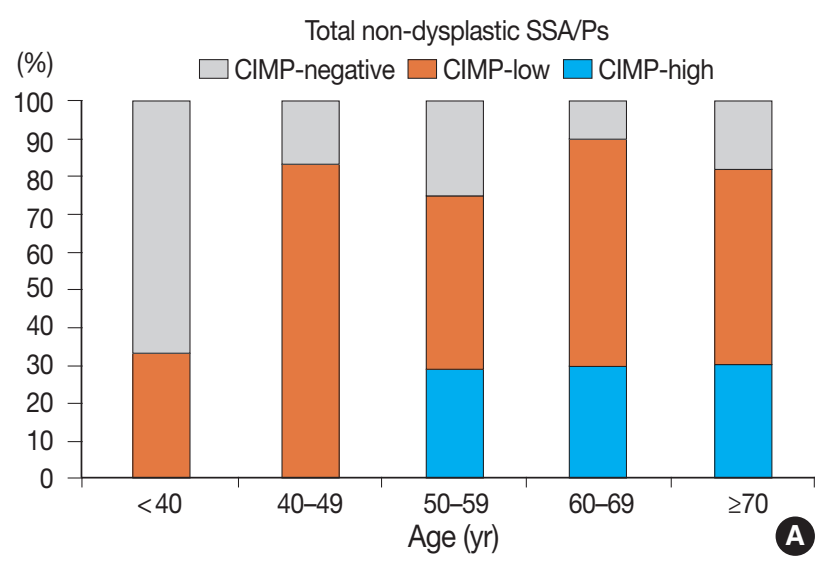

High-risk SSA/Ps (CIMP-high or MLH1-methylated SSA/PS)
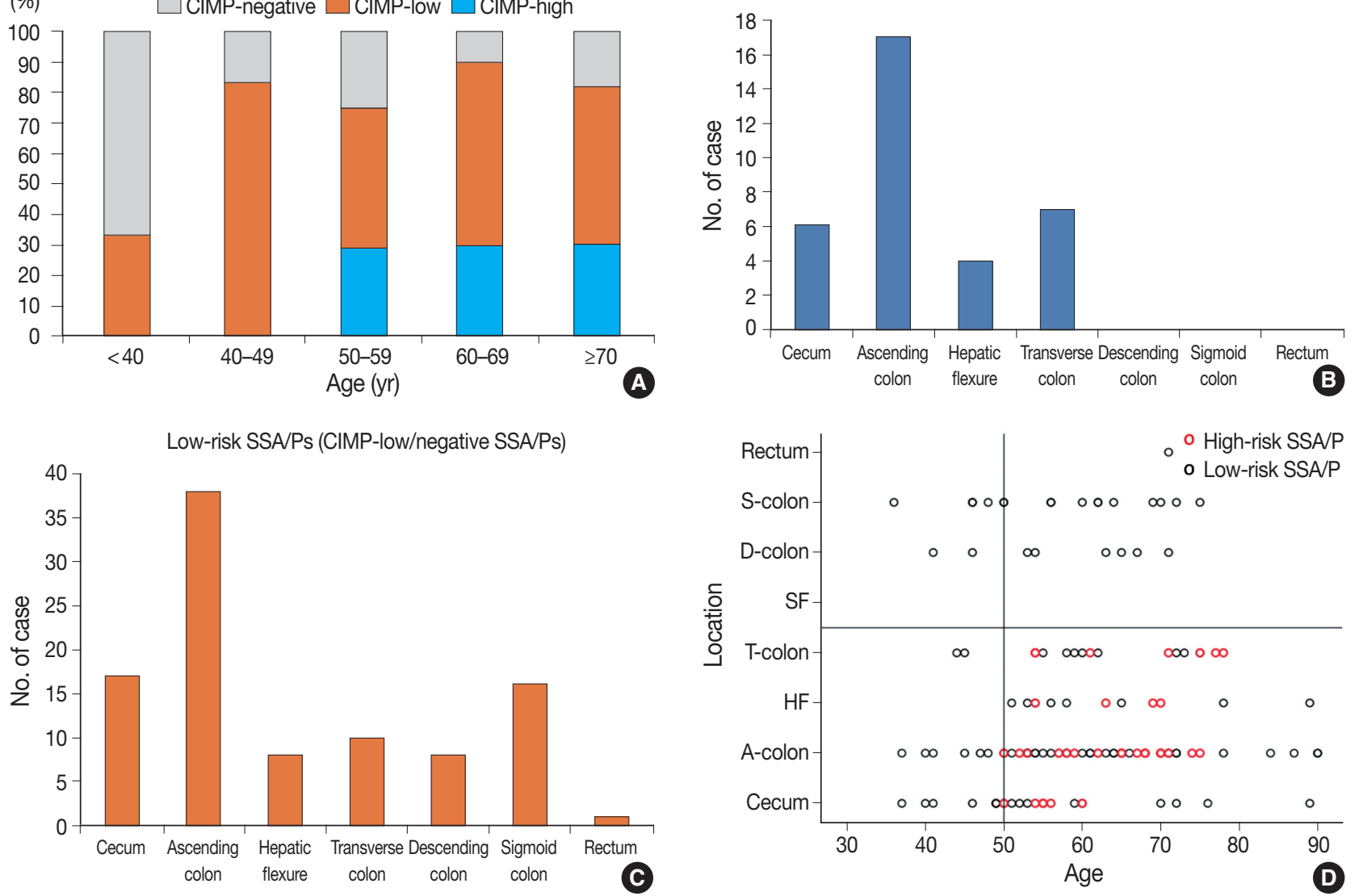

Fig. 3. The high-risk subgroup of non-dysplastic sessile serrated adenomas/polyps (SSA/Ps) is characterized by old age and proximal coIonic location. (A) Percentages of CpG island methylator phenotype (CIMP)-high, -low, and -negative subsets of non-dysplastic SSA/Ps in the different age subgroups. Note the presence of CIMP-high cases only in $\geq 50$ years age subgroups. (B) Frequencies of high-risk SSA/Ps according to the lesion location bowel subsites. Note the presence of high-risk SSA/Ps only in the proximal colon. (C) Frequencies of lowrisk SSA/Ps according to the lesion location bowel subsites. (D) Two-dimensional (age-location) scatter plot of 132 non-dysplastic SSA/Ps. S-colon, sigmoid colon; D-colon, descending colon; SF, splenic flexure; T-colon, transverse colon; HF, hepatic flexure; A-colon, ascending colon.

location of SSA/Ps, have been suggested by experts, based mainly on less-than-robust, inconclusive clinicopathologic data. ${ }^{6,10}$ Moreover, the 'cutoff' values for the number and size of SSA/Ps for the prediction of high-risk lesions have not been clearly established. Therefore, there is a strong need to precisely identify clinicopathologic risk factors for carcinomatous change in SSA/Ps, especially in SSA/Ps without dysplasia. As no official grading or risk-stratifying system exists for non-dysplastic SSA/Ps, investigating the clinicopathologic factors significantly associated with a high-risk subgroup of non-dysplastic SSA/Ps would be a worthwhile study for clinical and pathologic practices.

To define a high-risk subgroup of non-dysplastic SSA/Ps, we used molecular criteria (CIMP-high and/or MLH1 promoter methylation), because the accumulation of $\mathrm{CpG}$ island methylation is known as a molecular hallmark of progression of SSA/Ps (Figs. 2B, 5). ${ }^{11,12}$ Based on this aspect, both CIMP-high and
MLH1 methylation in SSA/Ps indicate lesions that are epigenetically high-potential to develop into carcinoma. CIMP-high is known to be tightly associated with $M L H 1$ methylation in CRCs, and MLH1 has been used as one of the essential markers for determination of CIMP-high status in CRCs. ${ }^{15,22}$ MLH1 deficiency via the methylation of its gene promoter can induce high microsatellite instability (MSI-high) and subsequent genome-wide hypermutated status in the tumor cells of premalignant lesions such as SSA $/ \mathrm{Ps}^{8}{ }^{8}$ and the hypermutated premalignant lesions can rapidly progress into carcinomas. ${ }^{6,10,21}$

In our present study, a total of 132 non-dysplastic SSA/Ps were classified into 34 high-risk and 98 low-risk lesions based on the $\mathrm{CpG}$ island methylation criteria (CIMP-high and/or MLH1 methylation). We performed statistical analyses using various clinicopathologic factors and found three major factors for potential use as predictive factors for high-risk lesions of non-dysplastic 
Table 1. Differential clinicopathologic and molecular features between CpG island methylation-based risk subgroups of non-dysplastic SSA Ps $(n=132)$

\begin{tabular}{|c|c|c|c|}
\hline Variable & High-risk SSA/Ps & Low-risk SSA/Ps & $p$-value \\
\hline \multicolumn{4}{|l|}{ Age (yr) } \\
\hline Older $(\geq 50)$ & $34(100)$ & $77(79)$ & .003 \\
\hline Younger $(<50)$ & 0 & $21(21)$ & \\
\hline \multicolumn{4}{|l|}{ Sex } \\
\hline Male & $28(82)$ & $58(59)$ & .015 \\
\hline Female & $6(18)$ & $40(41)$ & \\
\hline \multicolumn{4}{|l|}{ Location } \\
\hline Proximal colon & $34(100)$ & $73(74)$ & .001 \\
\hline Distal colorectum & 0 & $25(26)$ & \\
\hline \multicolumn{4}{|l|}{ Lesion size (endoscopically-measured) (mm) } \\
\hline Larger $(\geq 5)$ & $34(100)$ & $91(93)$ & .19 \\
\hline Smaller $(<5)$ & 0 & $7(7)$ & \\
\hline \multicolumn{4}{|l|}{ Lesion size (histologically-measured) (mm) } \\
\hline Larger $(\geq 5)$ & $34(100)$ & $80(82)$ & .004 \\
\hline Smaller $(<5)$ & 0 & $18(18)$ & \\
\hline \multicolumn{4}{|l|}{ Multiplicity (total number of synchronous serrated lesions) } \\
\hline More ( $\geq 3$ serrated lesions) & $5(15)$ & $24(24)$ & .235 \\
\hline Less (<3 serrated lesions) & $29(85)$ & $74(76)$ & \\
\hline \multicolumn{4}{|l|}{ Number of typical base-dilated serrated crypts } \\
\hline More ( $\geq 3$ typical crypts) & $32(94)$ & $75(77)$ & .024 \\
\hline Less (<3 typical crypts) & $2(6)$ & $23(23)$ & \\
\hline \multicolumn{4}{|c|}{ Largest diameter of typical base-dilated serrated crypts $(\mu \mathrm{m})$} \\
\hline Larger ( $\geq 251)$ & $19(56)$ & $37(38)$ & .065 \\
\hline Smaller $(<251)$ & $15(44)$ & $61(62)$ & \\
\hline \multicolumn{4}{|l|}{ MLH1 promoter methylation } \\
\hline Methylated & $8(24)$ & 0 & $<.001$ \\
\hline Unmethylated & $26(76)$ & $98(100)$ & \\
\hline \multicolumn{4}{|l|}{ CACNA1G promoter methylation } \\
\hline Methylated & $31(91)$ & $21(21)$ & $<.001$ \\
\hline Unmethylated & $3(9)$ & $77(79)$ & \\
\hline \multicolumn{4}{|l|}{ SOCS1 promoter methylation } \\
\hline Methylated & $12(35)$ & $7(7)$ & $<.001$ \\
\hline Unmethylated & $22(65)$ & $91(93)$ & \\
\hline \multicolumn{4}{|l|}{ CRABP1 promoter methylation } \\
\hline Methylated & $34(100)$ & $47(48)$ & $<.001$ \\
\hline Unmethylated & 0 & $51(52)$ & \\
\hline \multicolumn{4}{|l|}{ RUNX3 promoter methylation } \\
\hline Methylated & $24(71)$ & $12(12)$ & $<.001$ \\
\hline Unmethylated & $10(29)$ & $86(88)$ & \\
\hline \multicolumn{4}{|l|}{ IGF2 promoter methylation } \\
\hline Methylated & $28(82)$ & $13(13)$ & $<.001$ \\
\hline Unmethylated & $6(18)$ & $85(87)$ & \\
\hline \multicolumn{4}{|l|}{ CDKN2A promoter methylation } \\
\hline Methylated & $28(82)$ & $27(28)$ & $<.001$ \\
\hline Unmethylated & $6(18)$ & $71(72)$ & \\
\hline \multicolumn{4}{|l|}{ NEUROG1 promoter methylation } \\
\hline Methylated & $33(97)$ & $45(46)$ & $<.001$ \\
\hline Unmethylated & $1(3)$ & $53(54)$ & \\
\hline \multicolumn{4}{|l|}{ LINE-1 methylation level } \\
\hline Relatively hypermethylated (average level $\geq 78.81$ ) & $21(62)$ & $48(49)$ & .198 \\
\hline Relatively hypomethylated (average level < 78.81) & $13(38)$ & $50(51)$ & \\
\hline
\end{tabular}

Values are presented as number (\%).

SSA/P, sessile serrated adenoma/polyp; LINE-1, long interspersed nucleotide element-1. 

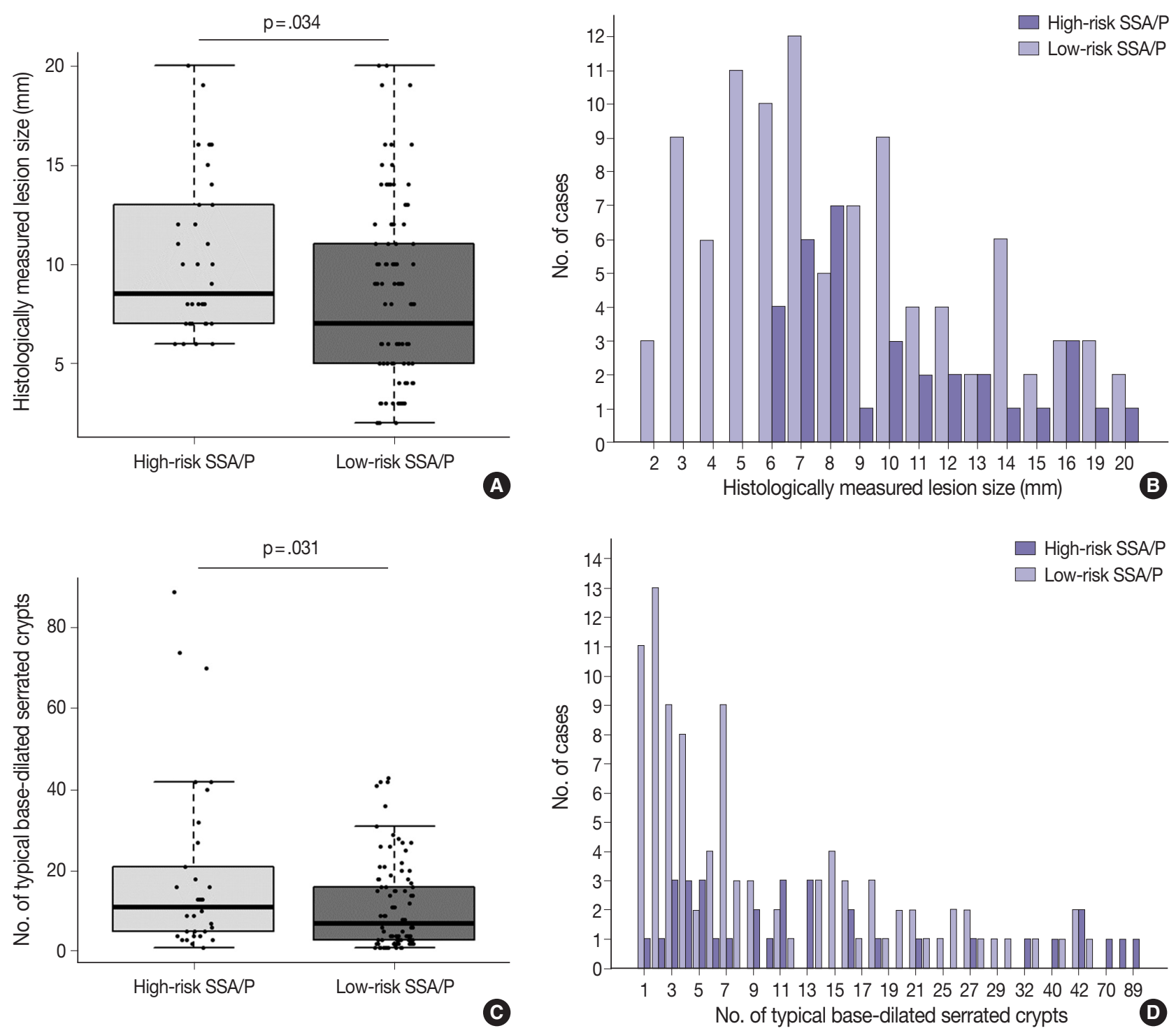

Fig. 4. Differences in histologically measured lesion size and number of typical base-dilated serrated crypts between high-risk and low-risk subgroups of 132 non-dysplastic sessile serrated adenomas/polyps (SSA/Ps). (A) A box-whisker-scatter plot for histologically measured lesion size. The p-value was obtained using the Student's t test. (B) Frequencies of high-risk SSA/Ps and low-risk SSA/Ps according to histologically measured lesion size. (C) A box-whisker-scatter plot for number of typical base-dilated serrated crypts. The p-value was obtained using the Mann-Whitney U test. (D) Frequencies of high-risk SSA/Ps and low-risk SSA/Ps according to number of typical base-dilated serrated crypts.

SSA/Ps: patient age, lesion location, and histologically measured lesion size (Fig. 5).

First, age is a significant factor for molecularly defined high-risk SSA/Ps. According to our results, the high-risk SSA/Ps were found exclusively in patients aged 50 years or more (Table 1, Fig. $3 \mathrm{~A}, \mathrm{D})$. This finding suggests that most of the SSA/Ps found in individuals under the age of 50 may be molecularly benign lesions, with a low risk of progression into malignancy. In fact, the present as well as previous studies suggest that age is important for understanding the characteristics of $\mathrm{CpG}$ island methylation in
SSA/Ps. Liu et al. ${ }^{23}$ recently published data highlighting the importance of age as a risk factor for CIMP-high and malignant progression in SSA/Ps. ${ }^{24}$ According to these studies, SSA/Ps in young patients rarely demonstrate CIMP-positive (CIMP-high) status, indicating a limited risk of malignant change in SSA/Ps of young patients. ${ }^{23,24}$ These results are very similar to our data. If consistent data are accumulated in future studies as well, it would strengthen the case for using age as an important risk-predictive factor in surveillance and diagnostic guidelines for SSA/Ps.

Our study also revealed that the lesion location could be a sig- 


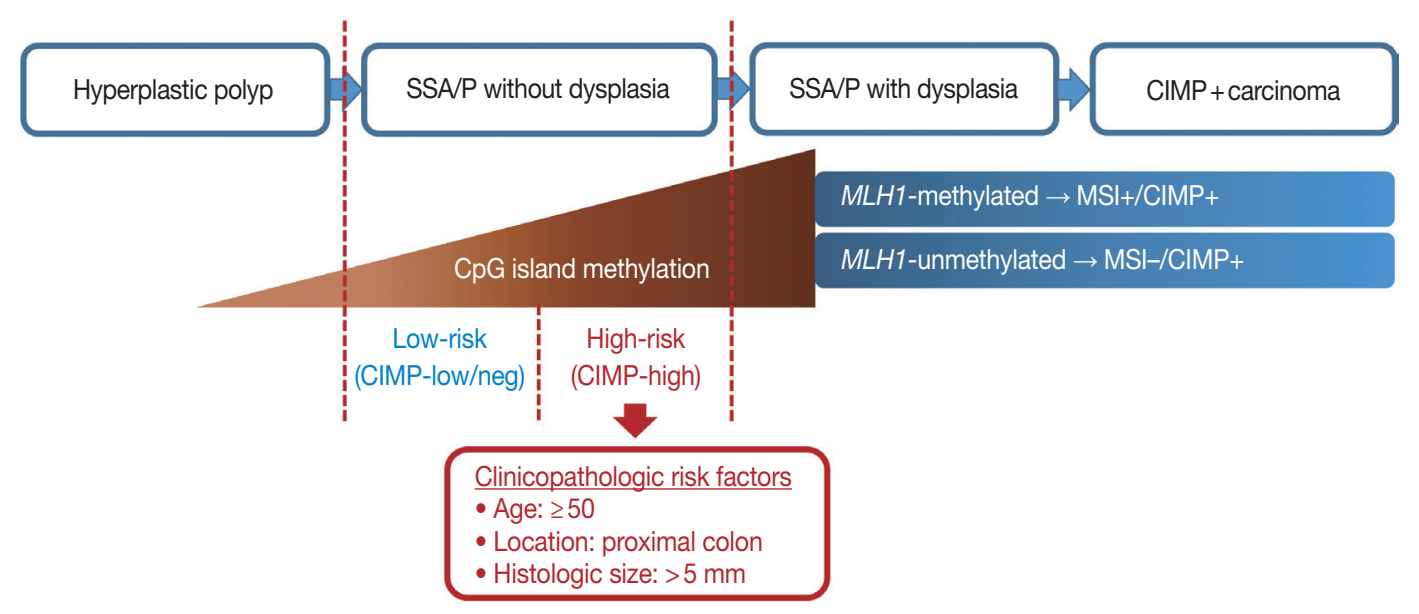

Fig. 5. A graphical summary of this study. Age, location, and size are potential predictive factors for a high-risk subgroup of non-dysplastic sessile serrated adenomas/polyps (SSA/Ps). CIMP, CpG island methylator phenotype; MSI, microsatellite instability.

nificant factor for the high-risk subgroup of non-dysplastic SSA/ Ps. All 34 high-risk SSA/Ps were located exclusively in the proximal colon, including cecum, ascending colon, hepatic flexure, and transverse colon (Table 1, Fig. 3B, D). This finding is not surprising, as previous studies have also suggested that the proximal colonic location is important for malignancy risk of SSA/Ps. ${ }^{6,10}$ The recent study by Liu et al..$^{23}$ found that CIMPhigh was significantly correlated with proximal location in SSA/ Ps, although a small number of distal-located SSA/Ps were also determined to be CIMP-high lesions. Through our results, the importance of lesion location in the prediction of malignant potential of SSA/Ps has been further confirmed.

In addition to patient age and lesion location, histologically measured lesion size was also a significant factor for high-risk SSA/Ps without dysplasia. All 34 high-risk SSA/Ps measured at least $6 \mathrm{~mm}$ in size, and the differences of histologically measured sizes between high-risk and low-risk SSA/Ps were statistically significant (Table 1, Fig. 4A, B). Notably, lesion size measured by endoscopy was not a statistically significant factor for the discrimination of high-risk lesions from low-risk SSA/Ps (Table 1, Supplementary Fig. S6). Although, similar to histologically measured lesion size, the endoscopically measured lesion size of all 34 high-risk SSA/Ps was at least $5 \mathrm{~mm}$, the majority of low-risk SSA/Ps (93\%) also demonstrated a size of $5 \mathrm{~mm}$ or more, when measured by endoscopy (Table 1), which likely led to the lack of statistical significance. These findings collectively indicate that histologically measured lesion size may be more valuable and crucial for the risk evaluation of SSA/Ps than endoscopically measured size. The superior value of lesion size measured by histomorphologic methods in the prediction of high-risk SSA/Ps should be validated by multiple independent studies in the future.

As mentioned earlier, although the multiplicity of serrated lesions has been regarded as a potential risk factor for malignant progression of SSA $/ \mathrm{Ps},{ }^{6}$ the increased number of synchronous serrated lesions was not a significant factor for high-risk nondysplastic SSA/Ps in our data (Table 1, Supplementary Fig. S3). In fact, because our study excluded cases satisfying diagnostic criteria for serrated polyposis, the expected high-risk lesions in serrated polyposis might be substantially removed from our study samples. As SSA/Ps in serrated polyposis might be clinicopathologically and molecularly different from sporadic SSA/ $\mathrm{Ps}^{5,25,26}$ we decided to focus on sporadically arising SSA/Ps in this study. Therefore, the value of multiplicity of serrated lesions in the prediction of high-risk SSA/Ps should not be underestimated based on the results of our study alone. Nevertheless, our data tend to suggest that multiplicity of serrated lesions may be less valuable than age, location, or size, for prediction of high-risk lesions among sporadic SSA/Ps.

In addition to the identification of clinicopathologic factors associated with the high-risk subgroup of non-dysplastic SSA/Ps, the elucidation of developmental pattern of $\mathrm{CpG}$ island methylation in SSA/Ps is another important finding from our study. Based on concurrently methylated marker-dependent and agedependent frequencies of methylation of each CIMP marker in 132 non-dysplastic SSA/Ps (Fig. 2A, Supplementary Fig. S1), we suggested a sequential model of methylation of CIMP markers in serrated neoplasia pathway (Fig. 2B). Early-methylated markers such as CRABP1 and NEUROG1 could be methylated in SSA/ Ps of young patients, whereas late-methylated markers such as SOCS1 and MLH1 could get methylated only in SSA/Ps of patients over the age of 50 (Supplementary Fig. S1). These results 
indicate that the accumulation of $\mathrm{CpG}$ island methylation during the progression of SSA/Ps is not a stochastic process, but rather an age-dependent, directed process. Our finding is valuable in understanding the characteristics of epigenetic alterations in colorectal carcinogenesis, because currently, the detailed pattern of CIMP development in serrated neoplasia pathway is poorly understood.

Promoter $\mathrm{CpG}$ island methylation in $M L H 1$ gene is a critical step for the development of MSI-high/CIMP-high CRCs from SSA/Ps. ${ }^{8}$ When $M L H 1$ is methylated and downregulated in an SSA/P without dysplasia, MSI-high and hypermutated phenotype can be induced in the genome of the SSA/P, and the nondysplastic SSA/P can rapidly progress into dysplasia and carcinoma. ${ }^{6,10,21}$ Based on our results, it can be inferred that the following two conditions should be satisfied for the MLH1 gene to be methylated in non-dysplastic SSA/Ps: (1) other CIMP markers sufficiently methylated in an SSA/P (generally three or more methylated markers) (Fig. 2A), and (2) old age of the patient (generally $\geq 50$ years old) (Supplementary Fig. S1). We also found that both complete loss of MLH1 expression and morphologic dysplasia may occur in SSA/Ps, when MLH1 promoter CpG islands are sufficiently methylated (Supplementary Table S1). MLH1 methylations in non-dysplastic SSA/Ps are mainly subrepressive alterations, because all $M L H 1$-methylated non-dysplastic SSA/Ps demonstrated relatively low PMR values and did not show a complete loss of MLH1 expression (Supplementary Table S1, Supplementary Fig. S2). Thus, MLH1 IHC may not be useful for the screening of high-risk lesions among non-dysplastic SSA/Ps.

In conclusion, $\mathrm{CpG}$ island methylation may be an age-dependent stepwise process in the colorectal serrated neoplasia pathway. Both CIMP-high and MLH1 methylation are late-step alterations during the progression of SSA/Ps and are unlikely to occur in SSA/ Ps of young patients. Proximal colon-located, $>5 \mathrm{~mm}$-sized SSA/Ps found in individuals aged $\geq 50$ should be considered as potential high-risk lesions, regardless of morphologic dysplasia or lesion multiplicity (Fig. 5).

\section{Electronic Supplementary Material}

Supplementary materials are available at Journal of Pathology and Translational Medicine (https://jpatholtm.org).

\section{ORCID}

Ji Ae Lee: https://orcid.org/0000-0003-1493-6208

Hye Eun Park: https://orcid.org/0000-0003-1048-2827
Seung-Yeon Yoo: https://orcid.org/0000-0002-7881-9038

Seorin Jeong: https://orcid.org/0000-0001-8448-1611

Nam-Yun Cho: https://orcid.org/0000-0002-2126-9549

Gyeong Hoon Kang: https://orcid.org/0000-0003-2380-6675

Jung Ho Kim: https://orcid.org/0000-0002-6031-3629

\section{Author Contributions}

Conceptualization: JHK.

Formal analysis: JAL, JHK.

Funding acquisition: JHK.

Investigation: JAL, HEP, SYY, SJ, NYC, JHK.

Methodology: JAL, HEP, SJ, NYC, JHK.

Project administration: JAL, HEP, JHK.

Resources: JAL, HEP, SYY, SJ.

Supervision: GHK, JHK.

Validation: NYC, JHK.

Visualization: JAL, JHK.

Writing—original draft: JAL, JHK.

Writing—review \& editing: GHK, JHK.

\section{Conflicts of Interest}

The authors declare that they have no potential conflicts of interest.

\section{Acknowledgments}

This study was supported by the grant from the SNUH Research Fund (04-2016-0680) and the National Research Foundation of Korea grant funded by the Korea government (Ministry of Science and ICT) (NRF-2016R1C1B2010627).

\section{REFERENCES}

1. Bae JM, Kim JH, Kang GH. Molecular subtypes of colorectal cancer and their clinicopathologic features, with an emphasis on the serrated neoplasia pathway. Arch Pathol Lab Med 2016; 140: 406-12.

2. Lochhead P, Chan AT, Giovannucci E, et al. Progress and opportunities in molecular pathological epidemiology of colorectal premalignant lesions. Am J Gastroenterol 2014; 109: 1205-14.

3. Snover DC. Update on the serrated pathway to colorectal carcinoma. Hum Pathol 2011; 42: 1-10.

4. Batts KP. The pathology of serrated colorectal neoplasia: practical answers for common questions. Mod Pathol 2015; 28 Suppl 1: S80-7.

5. Bosman FT, Carneiro F, Hruban RH, Theise ND. WHO classification of tumours of the digestive system. 4th ed. Lyon: International Agency for Research on Cancer, 2010.

6. Rex DK, Ahnen DJ, Baron JA, et al. Serrated lesions of the colorec- 
tum: review and recommendations from an expert panel. Am J Gastroenterol 2012; 107: 1315-29.

7. IJspeert JE, Vermeulen L, Meijer GA, Dekker E. Serrated neoplasiarole in colorectal carcinogenesis and clinical implications. Nat Rev Gastroenterol Hepatol 2015; 12: 401-9.

8. Kim JH, Bae JM, Cho NY, Kang GH. Distinct features between MLH1-methylated and unmethylated colorectal carcinomas with the $\mathrm{CpG}$ island methylator phenotype: implications in the serrated neoplasia pathway. Oncotarget 2016; 7: 14095-111.

9. Bateman AC, Shepherd NA. UK guidance for the pathological reporting of serrated lesions of the colorectum. J Clin Pathol 2015; 68: 585-91.

10. Crockett SD, Snover DC, Ahnen DJ, Baron JA. Sessile serrated adenomas: an evidence-based guide to management. Clin Gastroenterol Hepatol 2015; 13: 11-26.e1.

11. Parker HR, Orjuela S, Martinho Oliveira A, et al. The proto CpG island methylator phenotype of sessile serrated adenomas/polyps. Epigenetics 2018; 13: 1088-105.

12. Sakai E, Nakajima A, Kaneda A. Accumulation of aberrant DNA methylation during colorectal cancer development. World J Gastroenterol 2014; 20: 978-87.

13. Bae JM, Kim JH, Oh HJ, et al. Downregulation of acetyl-CoA synthetase 2 is a metabolic hallmark of tumor progression and aggressiveness in colorectal carcinoma. Mod Pathol 2017; 30: 267-77.

14. Oh HJ, Kim JH, Bae JM, Kim HJ, Cho NY, Kang GH. Prognostic impact of Fusobacterium nucleatum depends on combined tumor location and microsatellite instability status in stage II/III colorectal cancers treated with adjuvant chemotherapy. J Pathol Transl Med 2019; 53: 40-9.

15. Ogino S, Cantor M, Kawasaki T, et al. CpG island methylator phenotype (CIMP) of colorectal cancer is best characterised by quantitative DNA methylation analysis and prospective cohort studies. Gut 2006; 55: 1000-6.

16. Ogino S, Kawasaki T, Kirkner GJ, Kraft P, Loda M, Fuchs CS. Evaluation of markers for $\mathrm{CpG}$ island methylator phenotype (CIMP) in colorectal cancer by a large population-based sample. J Mol Diagn 2007; 9: 305-14.

17. Nosho K, Irahara N, Shima K, et al. Comprehensive biostatistical analysis of $\mathrm{CpG}$ island methylator phenotype in colorectal cancer using a large population-based sample. PLoS One 2008; 3: e3698.

18. Kim JH, Shin SH, Kwon HJ, Cho NY, Kang GH. Prognostic implications of $\mathrm{CpG}$ island hypermethylator phenotype in colorectal cancers. Virchows Arch 2009; 455: 485-94.

19. Wen X, Jeong S, Kim Y, et al. Improved results of LINE-1 methylation analysis in formalin-fixed, paraffin-embedded tissues with the application of a heating step during the DNA extraction process. Clin Epigenetics 2017; 9: 1.

20. Bettington M, Walker N, Rosty C, et al. Clinicopathological and molecular features of sessile serrated adenomas with dysplasia or carcinoma. Gut 2017; 66: 97-106.

21. Burgess NG, Tutticci NJ, Pellise M, Bourke MJ. Sessile serrated adenomas/polyps with cytologic dysplasia: a triple threat for interval cancer. Gastrointest Endosc 2014; 80: 307-10.

22. Weisenberger DJ, Siegmund KD, Campan M, et al. CpG island methylator phenotype underlies sporadic microsatellite instability and is tightly associated with BRAF mutation in colorectal cancer. Nat Genet 2006; 38: 787-93.

23. Liu C, Bettington ML, Walker NI, et al. CpG island methylation in sessile serrated adenomas increases with age, indicating lower risk of malignancy in young patients. Gastroenterology 2018; 155: 13625.e2.

24. Bettington M, Brown I, Rosty C, et al. Sessile serrated adenomas in young patients may have limited risk of malignant progression. J Clin Gastroenterol 2019; 53: e113-6.

25. He EY, Wyld L, Sloane MA, Canfell K, Ward RL. The molecular characteristics of colonic neoplasms in serrated polyposis: a systematic review and meta-analysis. J Pathol Clin Res 2016; 2: 127-37.

26. Edelstein DL, Axilbund JE, Hylind LM, et al. Serrated polyposis: rapid and relentless development of colorectal neoplasia. Gut 2013; 62: 404-8. 